\title{
ESTUDO ANATÔMICO DA PAREDE ANTERIOR DO ABDOME EM CADÁVER E HÉRNIA DE SPIEGEL
}

\author{
ANATOMICAL STUDY OF THE VENTRAL ABDOMINAL WALL IN CADAVER \\ AND SPIGELIAN HERNIA
}

\author{
Pedro Luiz Squilacci Leme, TCBC-SP ${ }^{1}$ \\ Darcy Lisbão Moreira de Carvalho, ACBC-SP2 \\ Marcio Botter, ACBC-SP ${ }^{3}$ \\ Otto-Michael Pius Höhne ${ }^{4}$ \\ José Alberto Salinas ${ }^{4}$ \\ Arildo de Toledo Viana, TCBC-SP ${ }^{5}$
}

\begin{abstract}
RESUMO: Objetivo: Tentar correlacionar a hérnia de Spiegel com eventuais alterações anatômicas da parede anterolateral do abdome. Abordar as particularidades do estudo anatômico em cadáver, com destaque aos músculos oblíquo interno, transverso abdominal, aponeurose de Spiegel, linha semilunar e ao aparecimento de hérnias de Spiegel. Método: A parede anterolateral do abdome foi dissecada em 31 cadáveres frescos do Departamento de Patologia da Santa Casa de Misericórdia de São Paulo, sendo realizada nos dois primeiros cadáveres a dissecção unilateral e nos 29 restantes o estudo bilateral da parede abdominal, completando 60 dissecções. Considerando que operamos no Hospital São Luiz Gonzaga, da Irmandade da Santa Casa de Misericórdia de São Paulo, 13 doentes com 14 hérnias de Spiegel, pudemos correlacionar os elementos clínicos aos estudos anatômicos em cadáver. Resultados: Defeitos encontrados nos músculos e aponeuroses: Oblíquo externo: 4/60 (6,6\%) - Oblíquo interno: 6/60 (10\%) - Transverso abdominal: 14/60 (23,3\%). Disposição dos músculos em forma de feixes de fibras: Oblíquo interno: 10/60 (16,6\%) - Transverso abdominal: 12/60 (20\%). Conclusões: As variações anatômicas e os defeitos encontrados, durante as dissecções do oblíquo interno e transverso abdominal, não se acompanharam de hérnias de Spiegel no cadáver, já a gordura pré-peritoneal, dissecando as fibras da aponeurose de Spiegel e oblíquo interno, foi encontrada nas operações e nas dissecções, podendo representar uma relação entre os defeitos musculoaponeuróticos da parede anterolateral do abdome e a hérnia de Spiegel.
\end{abstract}

Descritores: Estudo anatômico; Parede abdominal anterior; Hérnia de Spiegel.

\section{INTRODUÇÃO}

As hérnias de Spiegel são consideradas raras, embora desde a década de 1950 alguns autores as tratem como uma doença pouco reconhecida e não propriamente rara ${ }^{1}$. Com a ultra-sonografia e tomografia computadorizada houve um aumento de seus relatos após a década de $1980^{2}$. A literatu- ra latino-americana descreve poucas hérnias de Spiegel, sendo geralmente apresentados relatos de $\operatorname{casos}^{3,4}$. No Brasil, desde a década de 1980, encontramos relatos de três doentes no Rio de Janeiro 5, 6; um doente em Porto Alegre ${ }^{7}$; quatro doentes em São Paulo ${ }^{8}$ e um doente em Santa Catarina ${ }^{9}$.

Consideramos hérnia de Spiegel a protrusão de um saco peritoneal ou gordura pré-peritoneal, por um defeito

1. Professor Assistente, Faculdade de Ciências Médicas da Santa Casa de São Paulo

2. Mestre em Cirurgia, Faculdade de Ciências Médicas da Santa Casa de São Paulo

3. Pós-graduando em Cirurgia, Faculdade de Ciências Médicas da Santa Casa de São Paulo

4. Chefe de Serviço de Cirurgia

5. Professor de Técnica Cirúrgica, Faculdade de Ciências Médicas da Santa Casa de São Paulo

Recebido em 31/01/2001

Aceito para publicação em 22/05/2001

Trabalho realizado no Departamento de Patologia da Santa Casa de São Paulo e Serviço de Cirurgia do Hospital São Luiz Gonzaga, da Irmandade da Santa Casa de Misericórdia de São Paulo. 
na região aponeurótica do transverso abdominal, situada entre a linha semilunar e a borda lateral do reto abdominal.

Realizamos o estudo de cadáveres frescos, que fornece material não fixado, semelhante em cor e textura aos tecidos vivos, visando à revisão da anatomia cirúrgica. Para tanto, a parede anterolateral do abdome foi dissecada em 31 cadáveres frescos, entre janeiro de 1993 e agosto de 1994. Este estudo teve o objetivo de tentar correlacionar a hérnia de Spiegel com eventuais alterações anatômicas da parede anterolateral do abdome.

Embora exista mais de uma definição para a linha semilunar e se considere que a fusão dos folhetos musculares e aponeuróticos no local se faz de forma pouco precisa, tornando-a pouco definida, seguimos a definição que a descreve como a linha que marca a transição entre músculo e aponeurose do transverso abdominal, uma linha curva de convexidade externa, formando um arco da reborda costal ao púbis ${ }^{2,10,11}$. A porção de aponeurose do transverso abdominal que fica entre a linha semilunar e a borda lateral da bainha do reto abdominal é chamada de aponeurose de Spiegel ${ }^{2,12}$.

São descritas variações congênitas ou adquiridas da estrutura anatômica da aponeurose de Spiegel como causa de hérnias na região ${ }^{11,13}$. Esta região ficaria numa zona de transição embriológica, onde os músculos seriam menos resistentes; também existem variações do transverso abdominal, como sua ausência ou fusão com o oblíquo interno ${ }^{10}$.

O músculo transverso abdominal é profundo em relação às seis últimas cartilagens costais e se interdigita com as fibras do diafragma; se vistos do interior do abdome parecem um só músculo. Rives ${ }^{14}$ descreve esta continuidade com o diafragma; na porção lateral são imbricados e inseparáveis; posteriormente às últimas costelas se unem por uma aponeurose comum. Suas fibras inferiores são paralelas ao oblíquo interno e sua aponeurose contribui para a bainha do reto abdominal. Os músculos transversos participam da expiração e se comportam como um único músculo, agindo na manutenção das vísceras abdominais e mantendo a pressão intra-abdominal, que aumenta quando estes músculos se contraem.

É importante não confundir a aponeurose posterior do transverso abdominal com a fáscia transversal. Lateralmente a fáscia transversal se separa facilmente da porção muscular do transverso abdominal. Medialmente, junto a sua porção aponeurótica, isto não acontece e ocorre a fusão da fáscia com as fibras aponeuróticas ${ }^{15}$.

Nervos, artérias e veias passam pela bainha posterior do reto abdominal, isolados ou não. Usualmente encontramos uma artéria pequena, um nervo e duas veias recobertas por gordura e tecido conectivo. Lateralmente os vasos e nervos perfuram a linha semilunar, percorrendo o plano entre o transverso abdominal e o oblíquo interno. Os estudos de Milloy et al. ${ }^{16}$ mostraram que $48 \%$ das artérias que deixavam a face posterior do reto abdominal, atravessavam a linha semilunar e seguiam pela musculatura lateral do abdome. Também foram encontrados três ou quatro ramos da artéria epigástrica inferior perfurando a linha semilunar, entre o transverso abdominal e o oblíquo interno.
Considerando que entre fevereiro de 1988 e dezembro de 1995, operamos no Hospital São Luiz Gonzaga, da Irmandade da Santa Casa de Misericórdia de São Paulo, 13 doentes com 14 hérnias de Spiegel, estudamos elementos clínicos, correlacionando com os estudos em cadáver. Neste artigo abordaremos apenas as particularidades do estudo anatômico realizado.

\section{MÉTODO}

A parede anterolateral do abdome foi dissecada em 31 cadáveres frescos do Departamento de Patologia da Santa Casa de Misericórdia de São Paulo, entre janeiro de 1993 e agosto de 1994, sendo realizada nos dois primeiros cadáveres a dissecção unilateral e nos 29 restantes o estudo bilateral da parede abdominal, completando 60 dissecções. Vinte e um cadáveres eram do sexo masculino e dez do sexo feminino. A menor idade foi 51 anos e a maior 84 anos, com média de 65 anos.

Só estudamos cadáveres com mais de 50 anos, faixa etária onde as hérnias de Spiegel ocorrem com maior freqüência. Foram excluídos os cadáveres com incisões abdominais recentes e hérnias incisionais, sendo permitida apenas a incisão mediana infra-umbilical antiga, presente em quatro cadáveres desta faixa etária encontrados no período do estudo.

Usamos a incisão mediana de necropsia do manúbrio esternal até abaixo da sínfise púbica, preservando um fragmento de pele com o umbigo em sua posição anatômica e uma incisão horizontal sobre o púbis, prolongada até a face lateral da raiz da coxa do lado a ser estudado em dois cadáveres e bilateralmente em 29 cadáveres. A pele e o tecido subcutâneo foram rebatidos lateralmente desde a reborda costal, expondo toda a porção muscular e aponeurótica da parede anterolateral do abdome e a região inguinal.

A bainha dos músculos retos foi aberta longitudinalmente da reborda costal ao púbis e o reto abdominal foi medido, dissecado, seccionado próximo ao púbis e rebatido superiormente, permitindo o estudo de sua bainha posterior, linha arqueada, fáscia transversal e peritônio parietal.

Lateralmente dissecamos e seccionamos os músculos oblíquo externo e interno para expor o transverso abdominal, estudando as porções musculares e aponeuróticas. Medimos a maior largura que encontramos da aponeurose de Spiegel, entre o plano umbilical e o plano das espinhas ilíacas anteriores superiores. Dissecamos a aponeurose de Spiegel (Figura 1), entre a linha semilunar e a bainha do reto abdominal, avaliando-a da linha arqueada até onde a porção muscular do transverso abdominal ficava sob o reto abdominal.

Seccionamos a aponeurose de Spiegel da linha arqueada até seu limite superior, estudando os vasos sangüíneos e os nervos que a perfuravam. Avaliamos os vasos epigástricos inferiores profundos, os nervos da região (Figura 2) e possíveis áreas de enfraquecimento da parede abdominal, procurando hérnias. 


\section{RESULTADOS}

Realizamos 60 dissecções da parede abdominal. A ascite estava presente em nove (29\%) dos 31 cadáveres estudados; encontramos três hérnias umbilicais e uma hérnia epigástrica em nossas dissecções.

A linha semilunar foi estudada em todas as dissecções, sendo bem evidente. A aponeurose de Spiegel foi medida na região mais larga que encontramos, entre o plano umbilical e o plano das espinhas ilíacas anteriores superiores; esta medida variou de 1,5 a 3,5 centímetros, com média de 2,26 centímetros. Notamos um maior afastamento entre as fibras aponeuróticas em direção à região inguinal e tecido adiposo sobre esta aponeurose (Figura 1). Estes locais potenciais de fraqueza na musculatura justificariam o aparecimento de hérnias nesta localização.

Em todas as 60 dissecções, o músculo oblíquo externo era aponeurótico na localização da aponeurose de Spie-

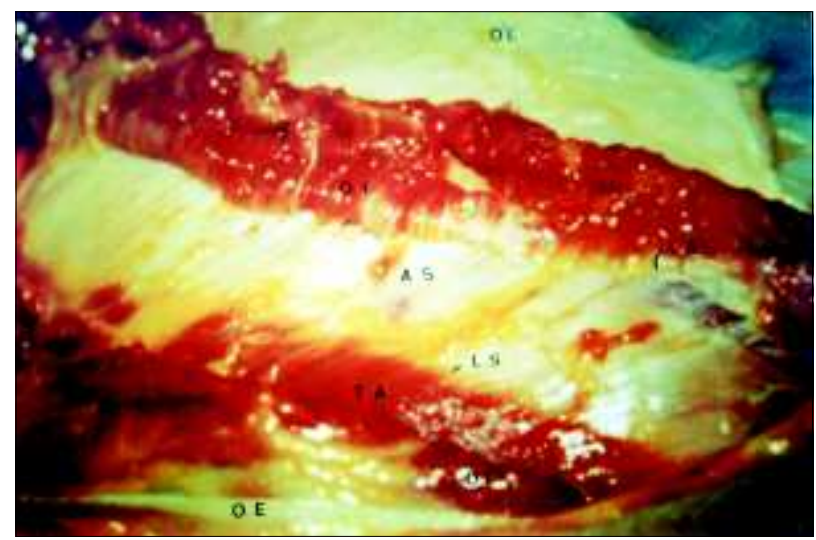

Figura 1 - Dissecção de cadáver - aponeurose de Spiegel à direita com tecido gorduroso entre as fibras aponeuróticas. OE: Oblíquo externo; OI: Oblíquo interno; LA: Linha arqueada; AS. Aponeurose de Spiegel; LS: Linha semilunar; TA: Transverso abdominal.

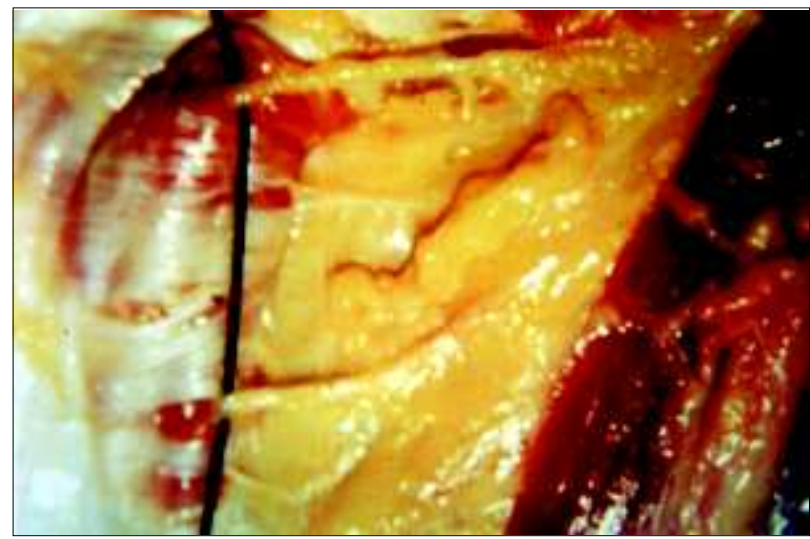

Figura 2 - Dissecção de cadáver - orifícios neurovasculares e defeitos fusiformes do transverso abdominal, abaixo da linha arqueada à esquerda. gel e em nenhuma delas sua porção muscular se localizou abaixo da linha das espinhas ilíacas anteriores superiores. Encontramos defeitos de sua porção aponeurótica (Tabela $1)$, com afastamento significativo de suas fibras, em quatro de 60 dissecções $(6,6 \%)$, porém nenhum defeito era percorrido por feixes neurovasculares. Nas dissecções este músculo se mostrou resistente e suas variações foram pouco significativas.

Quanto ao oblíquo interno, a disposição em forma de faixas formadas por feixes de fibras foi notada em dez dissecções $(16,6 \%)$, sendo notados defeitos fusiformes pouco pronunciados em seis dissecções $(10 \%)$. Não foi notado tecido adiposo em quantidade significativa neste folheto muscular, principalmente entre os feixes de fibras, quando estes existiram. Não caracterizamos pontos de fraqueza neste músculo e sua porção muscular cobria a aponeurose de Spiegel em todos os cadáveres estudados (Tabela 1).

O músculo oblíquo interno acessório foi encontrado bilateralmente em um cadáver $(3,3 \%)$.

Estudando o transverso abdominal, encontramos a disposição muscular em forma de feixes de fibras (Figura 3)

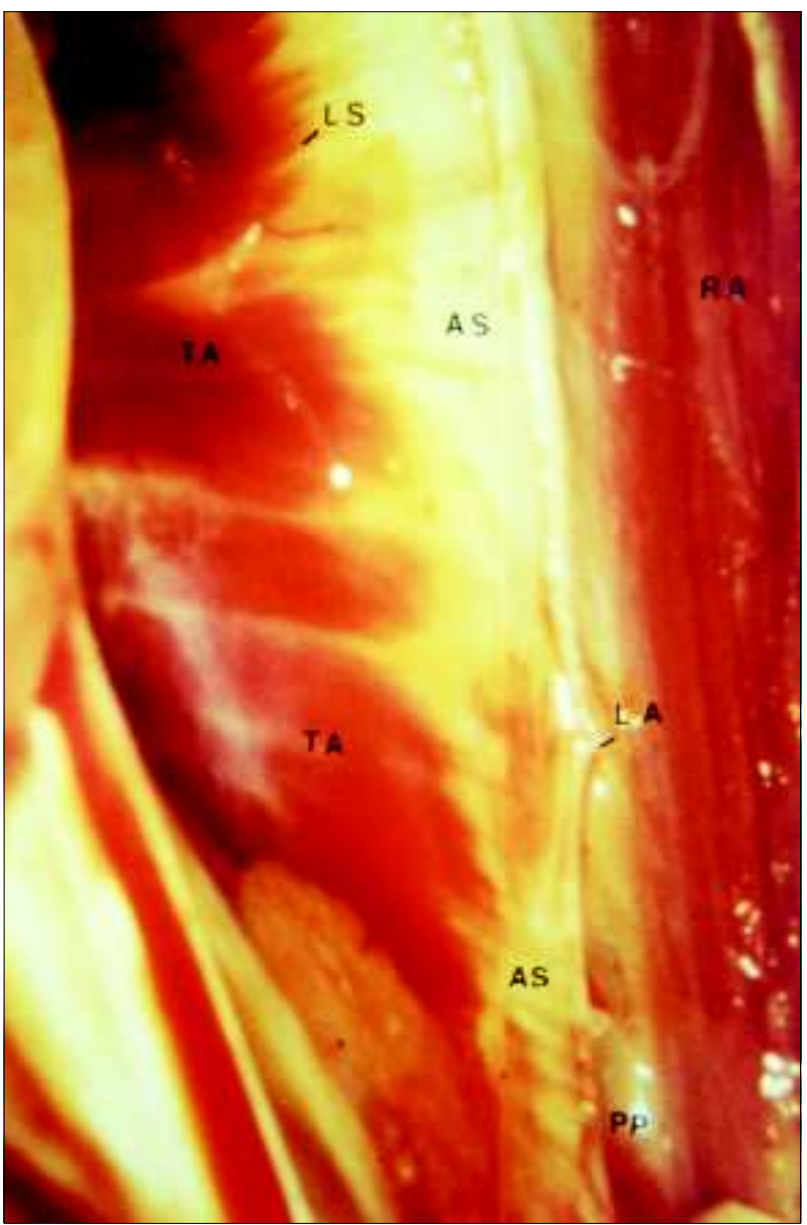

Figura 3 - Dissecção de cadáver - transverso abdominal com feixes de fibras em forma de faixas e tecido gorduroso entre as faixas. LS: Linha semilunar; TA: Transverso abdominal; AS: Aponeurose de Spiegel; RA: Reto abdominal; LA: Linha arqueada; PP: Peritônio parietal. 
Tabela 1

Características do oblíquo externo, oblíquo interno e transverso abdominal de 31 cadáveres dissecados

\begin{tabular}{l|c|c}
\hline Músculo dissecado & $\begin{array}{c}\text { Defeitos encontrados nos músculos } \\
\text { ou aponeuroses }\end{array}$ & $\begin{array}{c}\text { Disposição não usual, em forma } \\
\text { de feixes de fibras }\end{array}$ \\
\hline Oblíquo externo & $4 / 60(6,6 \%)$ & $10 / 60(16,6 \%)$ \\
Oblíquo interno & $6 / 60(10 \%)$ & $12 / 60(20 \%)$ \\
Transverso abdominal & $14 / 60(23,3 \%)$ & $(20)$ \\
\hline
\end{tabular}

em 12 dissecções (20\%). Além dos orifícios neurovasculares, vários defeitos fusiformes (Figura 2) foram encontrados na aponeurose do transverso abdominal em 14 dissecções $(23,3 \%)$. Pudemos evidenciar em todas as dissecções, grupos de fibras do transverso abdominal com os feixes de sua porção superior dirigindo-se superiormente e medialmente; os feixes de sua porção média dirigindo-se medialmente e os feixes de sua porção inferior dirigindo-se inferiormente e medialmente (Figura 3).

Em nenhuma das dissecções o transverso abdominal cobria a borda lateral da bainha do reto abdominal, não sendo encontrada nenhuma hérnia ou defeito na bainha posterior do reto nos cadáveres dissecados.

A linha arqueada (de Douglas) foi bem evidenciada em 39 dissecções (65\%), era pouco definida em dez dissecções $(16,6 \%)$, terminava gradualmente em nove dissecções (15\%), sendo ausente bilateralmente em um cadáver $(3,3 \%)$.

Determinamos a localização da linha arqueada em relação a dois planos constantes, o plano umbilical e o plano das espinhas ilíacas anteriores superiores. Encontramos, em 60 dissecções, 46 linhas arqueadas $(76,6 \%)$ entre o plano umbilical e a linha que une as espinhas ilíacas anteriores superiores; 12 linhas arqueadas (20\%) entre a linha das espinhas ilíacas e o púbis. Em um cadáver $(3,3 \%)$ a linha arqueada não foi encontrada bilateralmente.

Um ramo significativo da artéria epigástrica inferior profunda perfurava a bainha do reto abdominal em cinco dissecções $(8,3 \%)$, seguindo em direção ao transverso abdominal. Em todos os cadáveres encontramos quatro ou cinco orifícios neurovasculares (Figura 2) perfurando a aponeurose do transverso abdominal, próximo da borda lateral do reto abdominal, na região da aponeurose de Spiegel. Alguns eram alargados e com gordura no interior, mas sem hérnias nesta localização. Não foram encontrados defeitos ou hérnias na bainha posterior do reto abdominal nas 60 dissecções realizadas.

Encontramos variações do oblíquo interno e transverso abdominal, mas sem defeitos sobrepostos.

A disposição muscular em forma de faixas, formadas por feixes de fibras, foi mais evidente no transverso abdominal (12 em 60), correspondendo a $20 \%$ das dissecções, do que no oblíquo interno (dez em 60), correspondendo a $16,6 \%$ das dissecções. Os defeitos também foram mais evidentes no transverso abdominal (14 - 23,3\%), do que no oblíquo interno (seis - 10\%). Estes músculos se mostraram bastante imbricados e de difícil separação na região da aponeurose de Spiegel em todas as dissecções.

\section{DISCUSSÃO}

Não há uma explicação satisfatória para os defeitos na linha semilunar e este tipo de hérnia. Embora seja sugerida a etiologia congênita, a estrutura muscular e aponeurótica do transverso abdominal é considerada importante na etiologia das hérnias de Spiegel, que seriam adquiridas.

A pressão intra-abdominal é maior na porção inferior da cavidade abdominal e abaixo do umbigo as fibras do oblíquo interno e transverso abdominal são mais paralelas ${ }^{17}$, podendo haver um enfraquecimento da parede abdominal ${ }^{18}$.

Os estudos em cadáver e as cirurgias de Spangen ${ }^{11}$ reforçam estes dados. Este autor, revisando 979 hérnias de Spiegel relatadas, encontrou o registro de 32 localizadas acima do umbigo ${ }^{2}$.

Não se considera a obesidade fator significativo para o desenvolvimento destas hérnias, mas é valorizado o bom desenvolvimento muscular como fator importante na redução do risco de aparecimento das hérnias de Spiegel ${ }^{11}$.

A gordura pré-peritonial já foi citada como fator etiológico $^{19}$, pois dissecaria as fibras aponeuróticas e abriria caminho para a hérnia. Spangen ${ }^{1}$ encontrou uma hérnia constituída só de gordura pré-peritoneal, nas outras hérnias ela envolvia os sacos herniários. Esta presença constante da gordura justifica sua possível contribuição para o desenvolvimento de hérnias na aponeurose de Spiegel.

Já se sugeriu a importância do enfraquecimento ou paralisia muscular da parede abdominal. A eletromiografia dos doentes com hérnias de Spiegel operados não encontrou quaisquer alterações que as justificassem ${ }^{11}$. As cicatrizes cirúrgicas abdominais também foram consideradas um fator predisponente, pois enfraqueceriam a aponeurose de Spiegel ${ }^{1}$.

São descritas variações anatômicas em todos os músculos da parede abdominal anterior e os diferentes arranjos da musculatura abdominal não são excepcionais, mas apenas variações da disposição convencional ${ }^{10,20}$.

Cooper ensinava que as hérnias poderiam aparecer pelas pequenas aberturas dos vasos e nervos. $\mathrm{O}$ alargamento das aberturas da aponeurose de Spiegel, pela passagem de nervos e ramos da artéria epigástrica inferior profunda, foi incluído como possível fator para o aparecimento destas hérnias, mas o feixe neurovascular neste caso deve estar ao lado da hérnia, achado só notado em uma das 28 hérnias operadas por Spangen ${ }^{11}$. 
Embora existam vários defeitos irregulares na aponeurose do transverso abdominal, principalmente em seu ponto de fusão com a do oblíquo interno e com freqüência apareça um ramo da artéria epigástrica inferior, se considera que este ramo arterial tem pouca importância na etiologia desta hérnia ${ }^{19}$, pois raramente estes vasos passam pelos defeitos herniários. Considera-se também que a gordura pré-peritoneal que recobre os sacos herniários é suprida por vasos microscópicos, reforçando os relatos dos feixes neurovasculares pelas aberturas da aponeurose de Spiegel. Encontramos orifícios neurovasculares alargados, mas sem gordura ou hérnias se fazendo por estes orifícios.

Em seu tratado clássico, Watson ${ }^{21}$ considera a junção da linha semilunar com a linha arqueada um ponto de fraqueza, por onde apareceriam as hérnias de Spiegel; esta afirmação não é mais aceita.

Askar ${ }^{22}$ não conseguiu identificar com precisão a linha arqueada. Neste estudo não a encontramos bilateralmente em um cadáver e a localizamos entre o plano umbilical e o plano das espinhas ilíacas anteriores superiores em 46 dissecções. Considerando que a localização da linha arqueada é altamente variável, suas relações com a linha que une as espinhas ilíacas anteriores superiores são consideradas um plano de referência mais constante que o plano umbilical ${ }^{11}$.

A aponeurose de Spiegel pode ser recoberta pela porção muscular ou aponeurótica do oblíquo interno. Spangen ${ }^{11}$ encontrou hérnias de Spiegel com maior freqüência nos doentes em que esta aponeurose era recoberta pela porção muscular do oblíquo interno (nove em 28 doentes). Quando o oblíquo interno que a recobre é aponeurótico, há fusão das aponeuroses e este músculo reforça a aponeurose de Spiegel, dificultando o aparecimento das hérnias. Esta fusão do oblíquo interno e transverso abdominal na bainha lateral do reto acima da linha arqueada ficou evidente em nossas dissecções.

Considera-se a maior largura da aponeurose de Spiegel abaixo do umbigo importante na etiologia desta hérnia $^{23}$. Spangen ${ }^{11}$ valoriza uma faixa de seis centímetros acima da linha que une as espinhas ilíacas anteriores superiores, onde esta aponeurose e a aponeurose do oblíquo interno são mais largas, esta largura não difere quando há ou não hérnias ou outros defeitos e é improvável que este seja um fator importante. Nossas dissecções mostraram uma aponeurose de Spiegel larga, mas sem defeitos significativos, terminando gradualmente entre o umbigo e a reborda costal.

As fibras da aponeurose de Spiegel podem ser separadas mais facilmente abaixo do plano umbilical, sendo mais afastadas umas das outras, assim como aparecem acúmulos de gordura sobre esta aponeurose. Em nosso estudo em cadáver, a largura da aponeurose de Spiegel, a disposição do oblíquo interno e transverso abdominal em forma de faixas musculares formados por feixes de fibras e o tecido adiposo sobre a aponeurose de Spiegel, embora presentes, não se acompanharam de hérnias na região.
Zimmerman et al. ${ }^{13}$, em 100 dissecções da região inguinal, encontraram defeitos em dez transversos abdominais na região ou abaixo da linha arqueada. Vinte músculos apresentavam características de faixas musculares com feixes divergentes de fibras.

Askar $^{22}$, estudando o transverso abdominal em 40 cadáveres frescos, notou que seus feixes musculares não são realmente transversos. Em 38 (95\%) os feixes musculares superiores se dirigiam superiormente e medialmente, os feixes mediais eram horizontais e os feixes inferiores paralelos ao ligamento inguinal. Em dois cadáveres (5\%) todos os feixes se dirigiam inferior e medialmente, sendo paralelos uns aos outros. Esta disposição é demonstrada na Figura 3. Este mesmo autor, estudando a decussação das fibras aponeuróticas dos músculos abdominais na linha alba, encontrou uma decussação simples das fibras do transverso abdominal em $30 \%$ e uma decussação tripla em $70 \%$ de suas dissecções.

Anson et $a l .{ }^{24}$ encontraram defeitos fusiformes do transverso abdominal em 13\% de 100 dissecções. Embora existam várias, a maior variação deste músculo se deveu às inserções da borda inferior de sua aponeurose, que pode ser encontrada desde uma posição alta na bainha do reto abdominal até na borda medial do anel femoral. Estes defeitos fusiformes foram encontrados em nossas dissecções, mas o músculo se mostrou bastante resistente e seus defeitos foram pequenos e pouco significativos. Estes mesmos autores encontraram um ramo da artéria epigástrica inferior enfraquecendo esta aponeurose em cinco de 58 dissecções, dado que não encontramos em nossas dissecções.

Existem variações da aponeurose do transverso abdominal na borda lateral da bainha do reto abdominal ${ }^{11,25}$, sendo evidenciados dois folhetos aponeuróticos do transverso abdominal nesta região, que poderiam explicar um enfraquecimento da aponeurose de Spiegel. Não conseguimos demonstrar esta disposição da aponeurose em nosso estudo.

O contorno e as inserções do transverso abdominal são mais complexos que os do oblíquo interno, pois suas extensões tendinosas se imbricam para formar a bainha do reto abdominal.

Na região da aponeurose de Spiegel, as fibras musculares do oblíquo interno e transverso abdominal são paralelas. Como River ${ }^{19}$ e Spangen ${ }^{11}$, encontramos o oblíquo interno e o transverso abdominal bastante imbricados próximo à linha arqueada, sendo difícil sua separação.

Zimmerman et al. ${ }^{13}$ encontraram defeitos em um ou dois desses folhetos musculares em $45 \%$ de suas dissecções e relatam que em sua porção inferior e medial estes músculos apresentam interdigitação de fibras, formando faixas aponeuróticas, com áreas de enfraquecimento, que poderiam ser locais potenciais para o aparecimento de hérnias. Descrevem a disposição em forma de faixas formadas por feixes de fibras do transverso abdominal e principalmente do oblíquo interno. Estes autores, estudando cem regiões pélvicas, encontraram defeitos no oblíquo interno e transverso abdominal em dez dissecções, sendo seis 
defeitos sobrepostos nos dois músculos e quatro defeitos do transverso abdominal, craniais aos defeitos do oblíquo interno.

O tecido adiposo com freqüência preenche os defeitos e, em alguns, a herniação do tecido adiposo pré-peritoneal, passa pelo transverso abdominal e ocupa os defeitos do oblíquo interno ${ }^{24}$.

Encontramos faixas musculares em forma de leque no oblíquo interno e transverso abdominal. Em nossas dissecções não evidenciamos defeitos sobrepostos, mas apenas a disposição em forma de faixas principalmente do transverso abdominal, com escasso tecido adiposo entre os fascículos musculares, caracterizando áreas significativas de enfraquecimento da musculatura. Também encontramos defeitos em forma de fendas na aponeurose do transverso abdominal.

As variações anatômicas e os defeitos encontrados, durante as dissecções do transverso abdominal e oblíquo interno, não se acompanharam de hérnias de Spiegel no cadáver, já a gordura pré-peritoneal, dissecando as fibras da aponeurose de Spiegel e oblíquo interno, foi encontrada nas operações e nas dissecções, podendo representar uma relação entre os defeitos musculoaponeuróticos da parede anterolateral do abdome e a hérnia de Spiegel.

\begin{abstract}
Background: The present anatomical study on cadavers has been undertaken together with examinations of patients operated, with the particular aim of investigating the transversus abdominis, internal oblique muscles, Spigelian aponeurosis, semilunar line and the occurrence of Spigelian hernias. Method: Ventral abdominal wall was dissected in thirty-one cadavers, being the first two a unilateral dissection and the other twenty-nine a bilateral study of the abdominal wall, totalling sixty dissections. Considering that we operated thirteen patients with forteen Spiegelian hernias at Hospital São Luiz Gonzaga, from Irmandade da Santa Casa de Misericórdia de São Paulo, we were able to study clinical elements and compare them to the cadaveric material. Results: Muscular and aponeurotic malformation: external oblique muscle: 4/60 (6,6\%) - internal oblique muscle: 6/60 (10\%) - transverse abdominal: 14/60 (23,3\%). Unusual muscular fibers arrangement: internal oblique muscle: 10/60 (16,6\%) - transverse abdominal: 12/60 (20\%). Conclusions: The anatomical variations in muscular defects of the cadaveric material didn't match up to Spigelian hernias. The preperitoneal fat found between the fibers of Spigelian aponeurosis and the internal oblique muscle both in the surgery and in the cadaveric material might stand for a relation between musculoaponeurotic defects and the Spigelian hernias.
\end{abstract}

Key Words: Anatomical study; Ventral abdominal wall; Spigelian hernia.

\title{
REFERÊNCIAS
}

1. Olson RO, Davis WC. Spigelian hernia: rare or obscure? Am. J. Surg., 1968, 116:842-6.

2. Spangen L. Spigelian hernia. In: Nyhus LM, Condon RE. Hernia. 4.ed. Philadelphia, Lippincott, 1995, pp.381-92.

3. Zanniello JMA, Pundyk C, Diéguez J. Hernia llamada de Spiegel: cuatro observaciones. Rev. Argent. Cir., 1983, 45:138-40.

4. Flumeri EN, Guida ML, Strauss LT et al. Hernia de Spiegel: nuestra experiencia en 8 casos. Rev. Venez. Cir., 1989, 42:143-5.

5. Peixoto HS, Silva JHT, Braga LJF et al. Hérnia de Spiegel. J. Bras. Med., 1983, 44:121-2.

6. Monteiro JA, Perrotta U, Saraiva PRN et al. Hérnia de Spiegel estrangulada. Rev. Bras. Cir., 1984, 74:309-12.

7. Fraga JCS, Benfica FS, Gaffré OA et al. Hérnia de Spiegel: relato de um caso e revisão de literatura. Rev. HCPA \& Fac. Med. Univ. Fed. Rio Gd.do Sul, 1985, 5:135-7.

8. Leme PLS, Soler WV, Terzian HG et al. Hérnia de Spiegel: relato de quatro casos. Rev. Col. Bras. Cir., 1990, 17:114-8.
9. Franzon O, Nitschke CAS, Acampora AJD et al. Hérnia de Spiegel: relato de caso. Arq. Catarin. Med., 1994, 23:5-8.

10. Testut L, Latarjet A. Musculos del abdomen. In: Tratado de anatomía humana. Barcelona, Salvat, 1951, pp.94088. v.1.

11. Spangen L. Spigelian hernia. Acta Chir. Scand., 1976, (suppl.) 462.

12. Spangen L. Spigelian hernia. World J. Surg., 1989, 13:57380.

13. Zimmerman LM, Anson BJ, Morgan EH et al. Ventral hernia due to normal banding of the abdominal muscles. Surg. Gynecol. Obstet., 1944, 78:535-40.

14. Rives JD. Anatomy of the attachments of the diaphragm:their relation to the problems of the surgery of diaphragmatic hernia. Ann. Surg., 1942, 115:745-55.

15. McVay CB, Anson BJ. Inguinal and femoral hernioplasty. Surg. Gynecol. Obstet., 1949, 88:473-85.

16. Milloy FJ, Anson BJ, McAfee DK. The rectus abdominis muscle and the epigastric arteries. Surg. Gynecol. Obstet., 1960, 110:292-302. 
17. Skandalakis JE, Skandalakis PN, Skandalakis LJ. Abdominal wall and hernias. In: Surgical anatomy and technique. New York, Springer-Verlag, 1995, pp.123-96.

18. Hertzer NR, Montie JE. Spigelian hernia: a review of the literature and report of three cases. Cleveland Clin. Quart., 1971, 38:13-8.

19. River LP. Spigelian hernia: spontaneous lateral ventral hernia through the semilunar line. Ann. Surg., 1942, 116:405-11.

20. Anson BJ, McVay CB. Inguinal hernia: I. the anatomy of the region. Surg. Gynecol. Obstet., 1938, 66:186-91.

21. Watson LF. Incisonal and ventral hernia. In: Hernia. 3.ed. St Louis, Mosby, 1948, pp.366-86.

22. Askar OM. Surgical anatomy of the aponeurotic expansions of the anterior abdominal wall. Ann.R. Coll. Surg. Engl., 1977, 59:313-21.

23. Bertelsen S. The surgical treatment of Spigelian hernia. Surg. Gynecol. Obstet., 1966, 122:567-72.

24. Anson BJ, Morgan EH, McVay CB. Surgical anatomy of the inguinal region based upon a study of 500 body halves. Surg. Gynecol. Obstet., 1960, 111:707-25.

25. McVay CB. Abdominal wall. In: Anson \& McVay Surgical anatomy. 6.ed. Philadelphia, Saunders, 1984, pp.484584 .
26. Leme PLS. Hérnia de Spiegel - Cirurgia e Anatomia: estudo de treze doentes e trinta e um cadáveres. Dissertação (Mestrado em Cirurgia Geral). São Paulo. Faculdade de Ciências Médicas da Santa Casa de São Paulo, 1996, $117 \mathrm{p}$.

Endereço para correspondência:

Pedro Luiz Squilacci Leme

Av. Nações Unidas, 561/ap. 52

09726-110 - São Bernardo do Campo - SP

E-mail: gorableme@uol.com.br 